Recepción: 16 / 07 / 2018

Aceptación: 22 / 09 / 2018

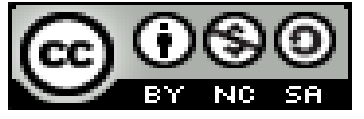

Ciencias sociales y políticas

Publicación: 01 / 11 / 2018

Artículo de investigación

\title{
Participación de mujeres y hombres en representaciones políticas del Ecuador
}

\section{Participação de mulheres e homens nas representações políticas do Equador \\ Participação de mulheres e homens nas representações políticas do Equador}

\author{
Natalia I. Zárate-Castro ${ }^{\mathrm{I}}$ \\ natalia.zarate@unl.edu.ec \\ Alexandra E. Aguilera-Maldonado II \\ alexandra.aguilera@unl.edu.ec \\ Dunia M. Yaguache-Maza III \\ dunia.yaguache@unl.edu.ec
}

\section{Correspondencia: natalia.zarate@hotmail.com}

\footnotetext{
${ }^{I}$ Magister en Desarrollo Comunitario, Licenciada en Ciencias de la Educación en la Especialidad de Químico

Biológicas, Profesora de Segunda educación en la Especialización de Químico Biológicas, Docente de la

Universidad Nacional de Loja, Loja, Ecuador.

II Magister en Ordenación Territorial, Arquitecto, Universidad Nacional de Loja, Loja, Ecuador.

III Magister en Gerencia Contable y Financiera, Doctora en Contabilidad y Auditoría, Licenciada en Contabilidad Y Auditoria-Contador Público-Auditor, Docente de la Universidad Nacional de Loja, Loja, Ecuador.
} 


\section{Resumen}

El estudio se desarrolla sobre la temática de la representación política y la participación de mujeres y hombres. La información para la formulación del presente análisis se obtuvo de las páginas oficiales del Instituto Nacional de Estadísticas y Censos (INEC), Consejo Nacional Electoral (CNE), Ministerio de Justicia Derechos, Humanos y Cultos. Y los documentos de revisión fueron: la Constitución de la República del Ecuador del 2008, Atlas Electoral del Ecuador 2009 -2014 realizado por el CNE, y el Atlas de Genero 2018 elaborado por el INEC. Se analiza la existencia de brechas en los espacios de representación política en el Ecuador desde lo nacional, provincial, cantonal hasta parroquial y el impacto de la equidad, para finalmente dejar planteando interrogantes relacionadas a las brechas que viven las mujeres en el ámbito de las representaciones en este caso político. Por lo que se considera para equiparar o disminuir la brecha existente en representaciones políticas y otras representaciones donde se da la toma de decisiones se debe fomentar y apoyar la participación social y política igualitaria de mujeres y hombres, con políticas públicas que permitan mejorar las condiciones de vida actuales y de futuras generaciones.

Palabras clave: representación política; paridad; empoderamiento; enfoque; brecha; alternabilidad.

\section{Abstract}

The study develops on the subject of political representation and the participation of women and men. The information for the formulation of the present analysis was obtained from the official pages of the National Institute of Statistics and Censuses (INEC), National Electoral Council (CNE), Ministry of Justice, Human Rights and Cults. And the revision documents were: The Constitution of the Republic of Ecuador of 2008, Electoral Atlas of Ecuador 2009 -2014 made by the CNE, and the Atlas of Gender 2018 prepared by the INEC. We analyze the existence of gaps in the areas of political representation in Ecuador from the national, provincial, cantonal to parochial and the impact of equity, to finally leave raising questions related to the gaps women live in the field of representations in this political case. For what is considered to equalize or diminish the existing gap in political representations and other representations where decisionmaking occurs, the equal social and political participation of women and men should be 
encouraged and supported, with public policies that allow improving the conditions of current life and future generations.

Key words: political representation; parity; empowerment; focus; gap; alternability.

\section{Resumo}

O estudo se desenvolve sobre o tema da representação política e a participação de mulheres e homens. A informação para a formulação desta análise foi obtida a partir do site oficial do Instituto Nacional de Estatística e Censos (INEC), Conselho Nacional Eleitoral (CNE), o Ministério dos Direitos da Justiça, Human e dos Assuntos Religiosos. E os documentos de revisão foram: a Constituição da República do Equador de 2008, o Atlas Eleitoral do Equador 2009 -2014 feito pela CNE, e o Atlas de Gênero 2018 elaborado pelo INEC. a existência de lacunas é analisado nas áreas de representação política no Equador a partir nacional, provincial, cantonal a freguesia eo impacto da equidade, para finalmente parar de levantar questões relacionadas com as lacunas que as mulheres experiência no campo das representações neste caso político. Por isso é considerado para corresponder ou diminuir a lacuna na representação política e outras representações em que a tomada de decisão é dada deve incentivar e apoiar a participação social e igualdade política entre homens e mulheres, com as políticas públicas para melhorar as condições vida atual e futuras gerações.

Palavras chave: representação política; paridade; empoderamento; foco violação alternabilidade.

\section{Introducción}

Ecuador tiene 16.624.858 habitantes; de los cuales 8.316.037 son mujeres lo que representa el $50,02 \%$ de la población del país y 8.308 .821 son hombres. (datosmacro.com, 2017). En la (Constitución de la República del Ecuador, 2008) artículo 116 establece que: Para las elecciones pluripersonales, la ley establecerá un sistema electoral conforme a los principios de proporcionalidad, igualdad del voto, equidad, paridad y alternabilidad entre mujeres y hombres; y determinará las circunscripciones electorales dentro y fuera del país. Consecutivamente, en el Plan Nacional de Desarrollo 2017-2021, en su objetivo 1, establece: Garantizar una vida digna con iguales oportunidades para todas las personas (SENPLADES, 2017). 
Según (Andaluza, Agencia) el Estado ecuatoriano, en su último informe oficial sobre la implementación de la CEDAW, reconoce que "ciertas prácticas jurídicas y sociales sí discriminan a las mujeres, tanto en la esfera pública como privada, pues tienen como efecto un tratamiento diferenciado a favor de los hombres en desventaja de las mujeres”. Según estudios de las instituciones estatales respectivas, en el Ecuador están legalizadas más de 1500 organizaciones de mujeres, las mismas que abordan una diversidad de ámbitos de acción como: la demanda por la igualdad en materia de derechos laborales, la lucha contra la discriminación en razón del género, la prevención de la violencia intrafamiliar, la participación paritaria en las diversas instancias de Gobierno, entre otras. Todas estas demandas han contribuido a la conformación de una gran diversidad de campos de acción en las que se ha ratificado a las mujeres como verdaderos actores en el ámbito político, social, cultural, entre otros ámbitos de trascendencia estatal. (Salazar V. P., 2017)

A pesar de los avances en las legislaciones de la región para superar las brechas de género, éstas persisten. Las mujeres aún tienen como pendiente en sus agendas el derecho a vivir libres de violencia en la casa, en las calles, en los parques, en los centros de estudio o de trabajo, en sus organizaciones políticas, en sus cargos de representación; las mujeres aún tienen que pelear por cambios en la norma su remuneración es menor que la de los hombres aunque hagan el mismo trabajo; aún tienen menos ingresos que los hombres porque sobre todo representan el segmento del mercado laboral menos pagado el de servicios, así como el subempleo y la informalidad; las mujeres aún trabajan 17h47 minutos más que los hombres cada semana; tienen menos acceso a/y control de los medios de producción, del capital y de su propio tiempo y, sin embargo, son las encargadas de garantizar el cuidado de las familias. Las mujeres siguen peleando por participar en igualdad de condiciones y con las mismas oportunidades que los hombres en la política, pero todos los retos pendientes arriban mencionados hacen que sea -a pesar de todos los logros y avances- una meta lejana; un sueño que se acaricia en la distancia, casi como un imposible. Según la Unión Interparlamentaria a Julio 2017, en el mundo, el 23.5\% de los escaños en las cámaras alta y baja combinadas, estaban ocupados por mujeres, mientras que en las Américas el 28.1\% de los escaños de las mismas cámaras estaban ocupados por mujeres (Aído, 2017).

Como se evidencia, existen ciertos avances en el establecimiento de normativa que pretende promover la representación paritaria de mujeres y hombres en la designación política y/o publica 
en los niveles donde se realiza la toma decisiones, sin embargo, como podemos observar los datos que se revelan en las elecciones del 2014 muestran lo contrario.

\section{Materiales y Métodos}

La obtención de la información básica implicó revisión de las bases de datos de las páginas oficiales de las siguientes instituciones: el Consejo Nacional Electoral, Ecuador en Cifras INEC, Ministerio de Justicia Derechos, Humanos y Cultos y otros. La revisión documental involucró información procedente de fuentes directas como es la Constitución de la República del Ecuador, que establece los principios de equidad de género; y fuentes indirectas como los resultados de las elecciones del 2014 publicados por el Consejo Nacional Electoral en el Atlas Electoral del Ecuador 2009 -2014; así también el Atlas de Genero 2018 elaborado por el INEC. La información estadística obtenida de fuentes directas e indirectas se representa en gráficos que ilustran o dan a conocer los datos cuantitativos, mismos que concluyen con la presentación de análisis cualitativos.

\section{Resultados y Discusión}

A continuación, se presenta información recabada de los resultados de las elecciones del 2014, publicados por el Consejo Nacional Electoral.

Grafico No 1: Autoridades Nacionales: Asambleístas Provinciales grado de participación.

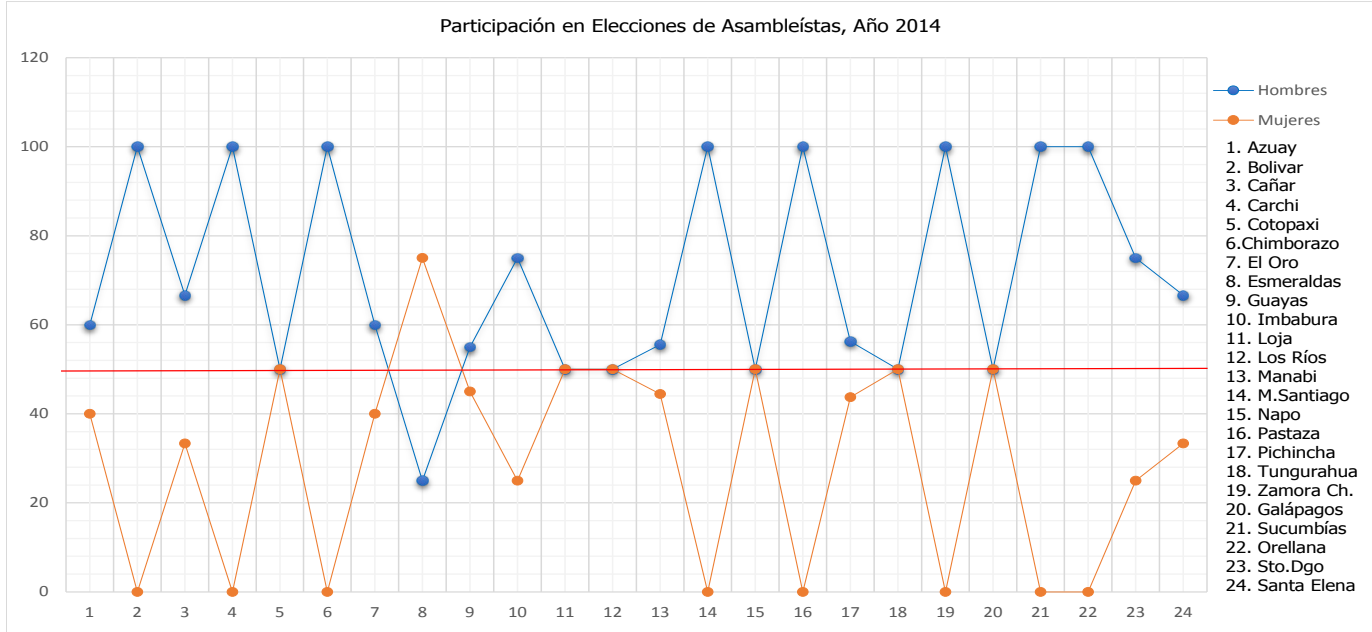

Fuente: Consejo Nacional Electoral - Elecciones 2014

Elaborado por: Autoras 
Grafico No 2: Autoridades Provinciales: Prefectos grado de participación.

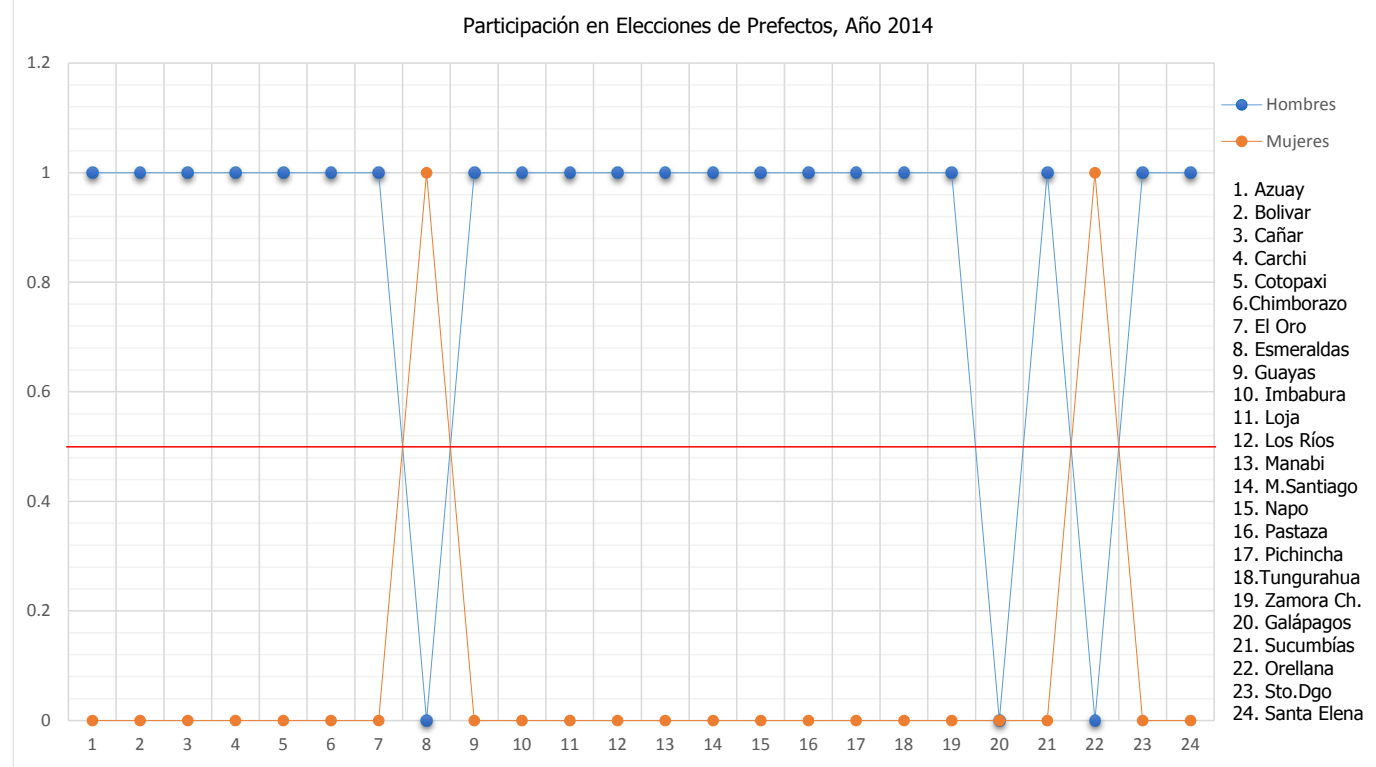

Fuente: Consejo Nacional Electoral - Elecciones 2014

\section{Elaborado por: Autoras}

Grafico No 3: Autoridades Cantonales: Alcaldías grado de participación.

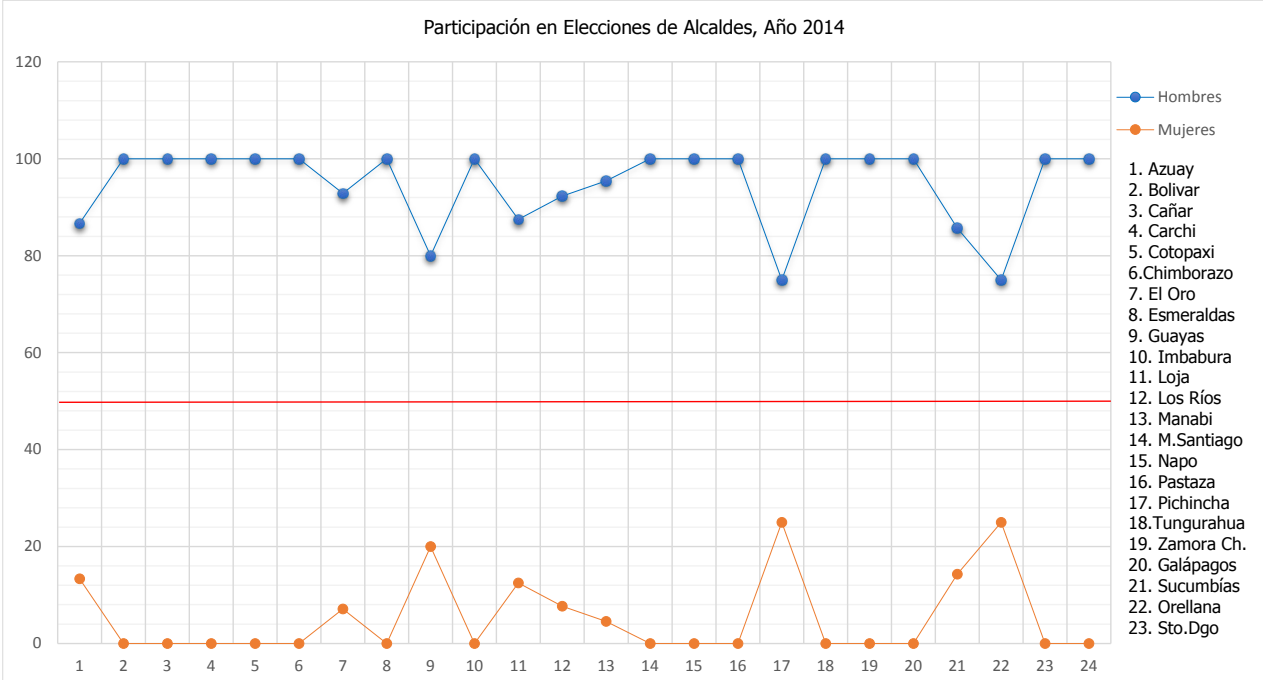

Fuente: Consejo Nacional Electoral - Elecciones 2014

Elaborado por: Autoras 
Grafico No 4: Autoridades Cantonales: Concejales Rurales grado de participación.

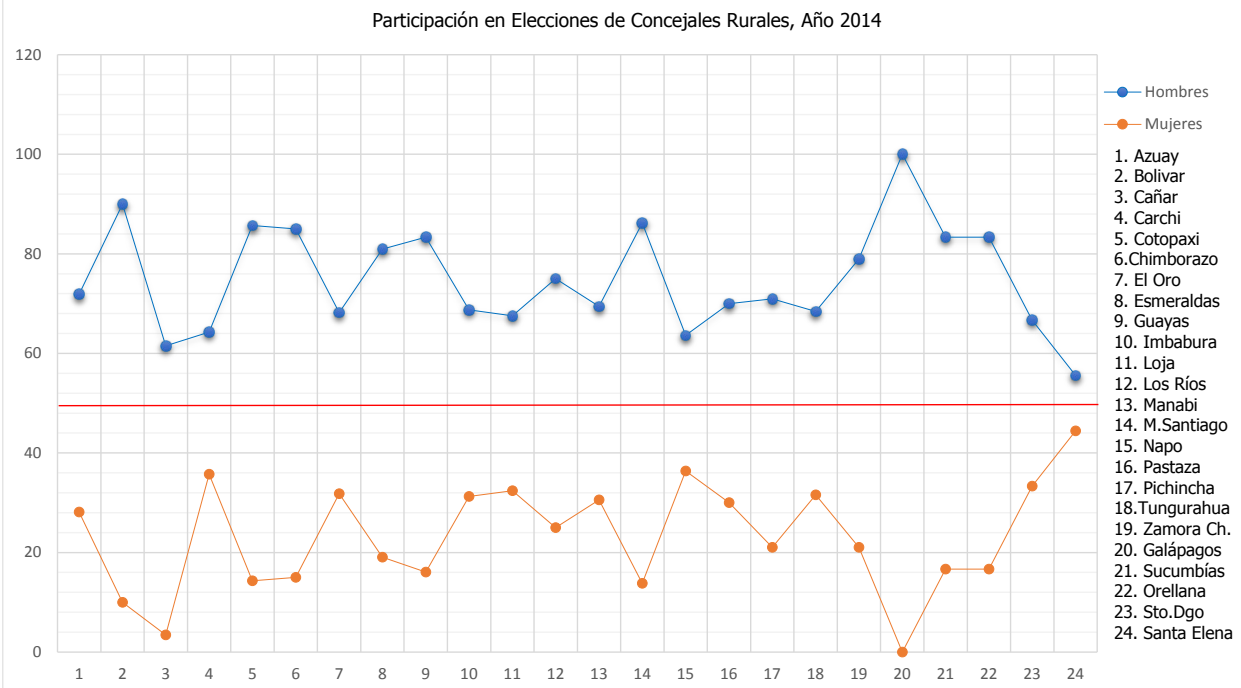

Fuente: Consejo Nacional Electoral - Elecciones 2014

Elaborado por: Autoras

Grafico No 5: Autoridades Cantonales: Concejales Urbanos grado de participación.

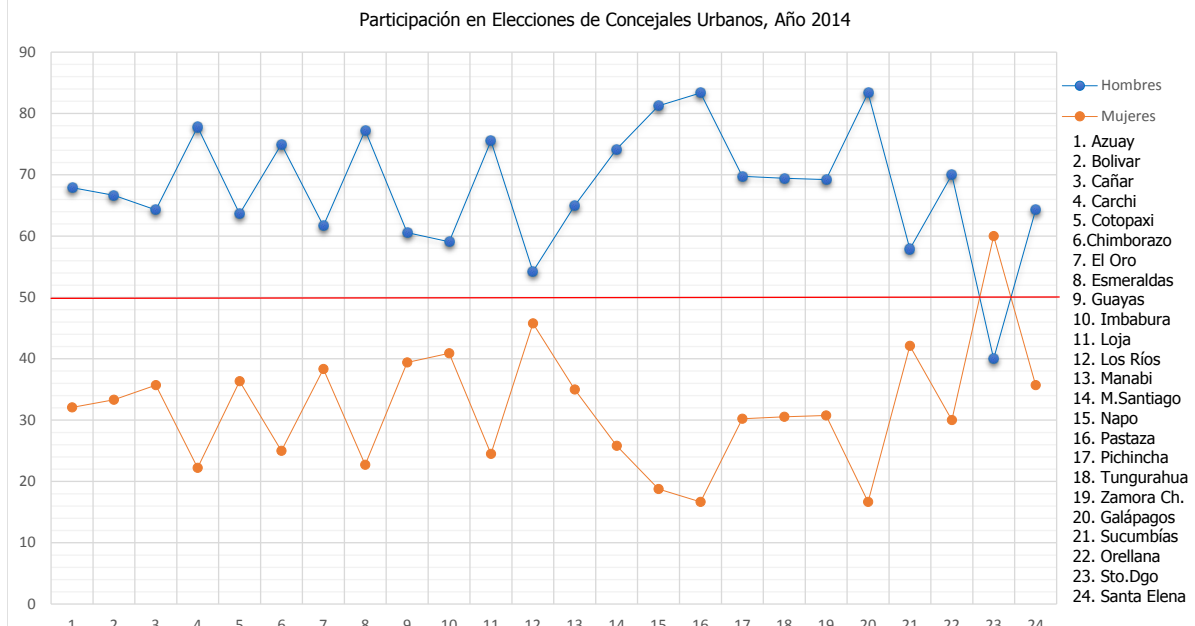

Fuente: Consejo Nacional Electoral - Elecciones 2014

Elaborado por: Autoras 
Grafico No 6: Autoridades Parroquiales: Vocales de Juntas Parroquiales Rurales grado de participación.

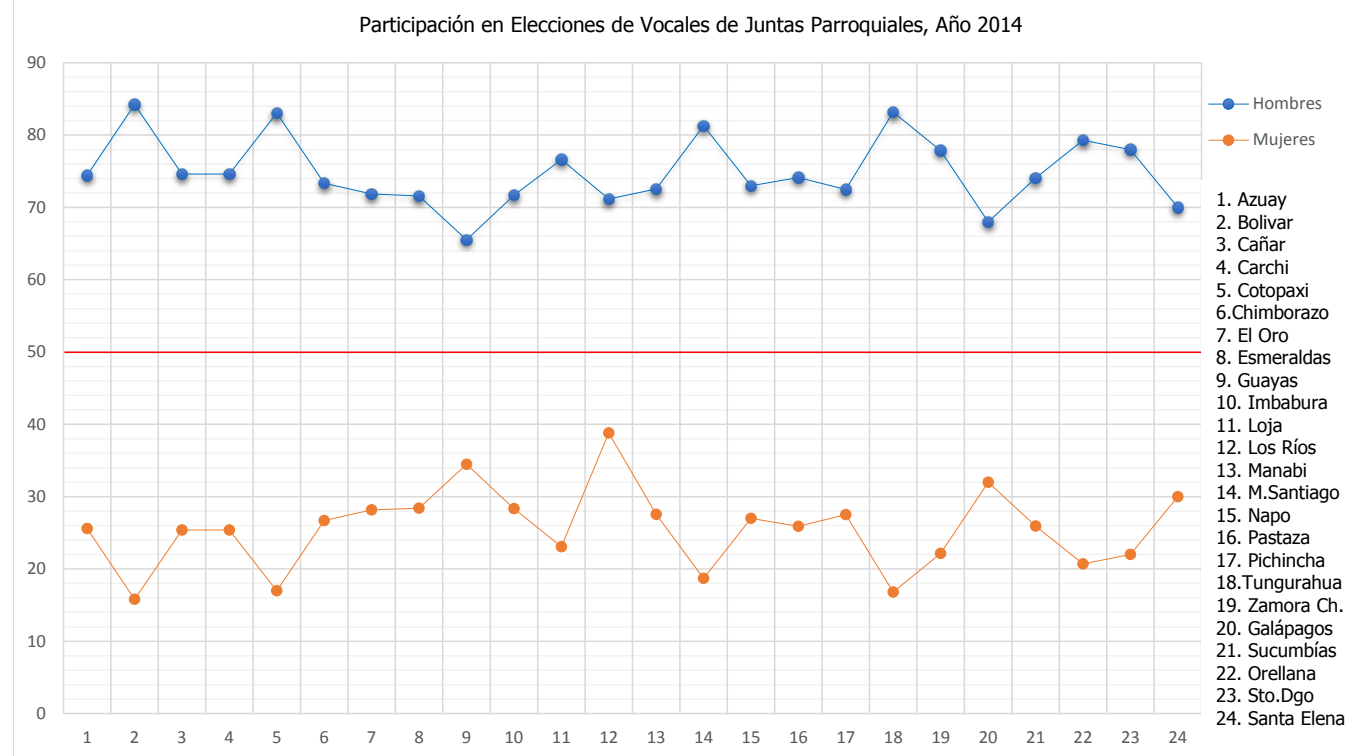

Fuente: Consejo Nacional Electoral - Elecciones 2014

\section{Elaborado por: Autoras}

Los resultados muestran de manare muy notable que no existe participación igualitaria en las elecciones del 2014, tanto a nivel de Asambleístas, Autoridades Provinciales, Autoridades Cantonales: Alcaldías, Concejales Rurales, Concejales Urbanos y Vocales de Juntas Parroquiales. Por lo que se considera que se debe brindar atención a la brecha existente en representaciones políticas y otras representaciones donde se da la toma de decisiones y con ello fomentar y apoyar la participación social y política igualitaria de mujeres y hombres, con políticas públicas que permitan mejorar las condiciones de vida actuales y de futuras generaciones. Por lo descrito se precisa analizar la definición de constitución del sujeto político que según (Salazar, 2013) manifiesta que existen dos segmentos sociales que pueden y deben constituirse en el nuevo sujeto político que irrumpa en el escenario de la sociedad contemporánea: los jóvenes y las mujeres. Las formas de pensar y hacer la política están fundamentadas en las colectividades, la fusión de fuerzas y la capacidad organizativa y movilizadora de estos dos segmentos. Por otra parte, desde la perspectiva formalista, (Pitkin, 1967) identifica una primera dimensión de la autorización que significa que "un representante es alguien que ha sido autorizado para actuar. Esto significa que 
le ha sido otorgado un derecho de actuar que no tenía anteriormente, mientras el representado se hace responsable de las consecuencias de esa acción como si la hubiera realizado él mismo". En ese contexto y como antecedente (Pescader, 2006) indica que, los problemas vinculados con la crisis de la representación que afronta el sistema político democrático en Argentina, y en muchos otros países latinoamericanos y del resto del mundo, nos acercan sólo a una dimensión de "lo político". Y por ello es relevante tener presente lo que manifiesta (Pescader, 2006) respecto de la representación política es una manera de hacer "visible" el poder político que refuerza un cierto tipo de dominación: la racional legal. El poder político es el elemento central de la dimensión político-institucional de una sociedad. Esta dimensión es la que distingue e identifica a los grupos humanos en la medida en que les permite organizar el mundo a partir de determinados principios: la racionalidad, la ética, la ley, la libertad, la solidaridad, la justicia, etc.; y darle sentido a la vida de la comunidad.

\section{Conclusiones}

En las elecciones del 2014, existe porcentajes mínimos de participación de las mujeres en las representaciones políticas, por lo que en los altos cargos de poder político donde se toman decisiones carecer de participación igualitaria, por ende, no estamos representadas a nivel local, provincial y nacional. Y por lo tanto las normas existentes no son suficientes deben aplicarse y mejorarse.

Existe una brecha acentuada en el ámbito de la participación de las mujeres en las representaciones políticas de alto nivel; ante tal afirmación se plantea la siguiente pregunta ¿Cómo el estado ecuatoriano está abordando tal problemática? ¿Cómo el estado ecuatoriano abordará y garantizará la paridad y alternabilidad entre mujeres y hombres?

\section{Referencias Bibliográficas}

En la Constitución de la República del Ecuador. (2008). Ecuador.

Acosta, A. (s.f.). En los Procesos económicos contemperanteos. Impacto de las reformas neoliberales en la economía. 
Aído, B. (10 de 2017). Opinión Electoral. Recuperado el 26 de 10 de 2018, de http:/ciespal.org/wp-content/uploads/2017/10/Serie-Gaceta-Opini-n-Electoral-No.19-Octubre2017.pdf

Andaluza, Agencia. (s.f.). Diagnóstico País Ecuador.

Consejo Nacional de Igualdad de Género. (2014). Agenda Nacional de las Mujeres y la Igualdad de Género.

En datosmacro.com. (2017). Expansión. Recuperado el 26 de 10 de 2018, de Población Ecuador: https://datosmacro.expansion.com/demografia/poblacion/ecuador

Elizondo, A., \& Vasco, U. d. (s.f.).

ONU. (2018). http://www.unwomen.org/es/how-we-work/intergovernmental-support/worldconferences-on-women.

Pescader, C. (2006). La representación política en cuestión. Fundamentos, características y dilemas.

Pitkin, H. F. (1967). El concepto de representación.

RESGE; Red de Educación Superior y Género del Ecuador. (2014). Calidad en la educación superior en América Latina. Quito.

Salazar, R. (2013). La construcción del Nuevo sujeto político en América Latina. Sociedad.

Salazar, V. P. (19 de 10 de 2017). Gaceta de Análisis Político Electoral. Recuperado el 26 de 10 de 2018, de http:/ciespal.org/wp-content/uploads/2017/10/Serie-Gaceta-Opini-n-ElectoralNo.19-Octubre-2017.pdf

SENPLADES, S. N. (2017). Plan Nacional de Desarrollo 2017-2021. 


\section{Anexos}

Tabla No 1: Autoridades Nacionales: Asambleístas Provinciales, desagregación por género

\begin{tabular}{|c|c|c|c|c|c|c|}
\hline \multirow{2}{*}{ Codigo } & \multirow[b]{2}{*}{ Provincia } & \multicolumn{5}{|c|}{ Asambleistas Provinciales } \\
\hline & & Hombres & MUJERES & TOTAL & $\%$ HOMBRES & \%MUJER \\
\hline 1 & AZUAY & 3 & 2 & 5 & 60 & 40 \\
\hline 2 & BOLIVAR & 3 & 0 & 3 & 100 & 0 \\
\hline 3 & CAÑAR & 2 & 1 & 3 & 66.67 & 33.33 \\
\hline 4 & CARCHI & 3 & 0 & 3 & 100 & 0 \\
\hline 5 & СОTOPAXI & 2 & 2 & 4 & 50 & 50 \\
\hline 6 & CHIMBORAZO & 4 & 0 & 4 & 100 & 0 \\
\hline & EL ORO & 3 & 2 & 5 & 60 & 40 \\
\hline & ESMERALDAS & 1 & 3 & 4 & 25 & 75 \\
\hline 9 & GUAYAS & 11 & 9 & 20 & 55 & 45 \\
\hline 10 & IMBABURA & 3 & 1 & 4 & 75 & 25 \\
\hline 11 & LOJA & 2 & 2 & 4 & 50 & 50 \\
\hline 12 & LOS RIOS & 3 & 3 & 6 & 50 & 50 \\
\hline 13 & MANABI & 5 & 4 & 9 & 55.56 & 44.44 \\
\hline 14 & MORONA SANTIAGO & 2 & 0 & 2 & 100 & 0 \\
\hline 15 & NAPO & 1 & 1 & 2 & 50 & 50 \\
\hline 16 & PASTAZA & 2 & 0 & 2 & 100 & 0 \\
\hline 17 & PINCHINCHA & 9 & 7 & 16 & 56.25 & 43.75 \\
\hline 18 & TUNGURAHUA & 2 & 2 & 4 & 50 & 50 \\
\hline 19 & ZAMORA CHINCHIPE & 2 & 0 & 2 & 100 & 0 \\
\hline 20 & GALAPAGOS & 1 & 1 & 2 & 50 & 50 \\
\hline 21 & SUCUMBIOS & 3 & 0 & 3 & 100 & 0 \\
\hline 22 & ORELLNA & 2 & 0 & 2 & 100 & 0 \\
\hline 23 & STO DGO TSACHILAS & 3 & 1 & 4 & 75 & 25 \\
\hline 24 & SANTA ELENA & 2 & 1 & 3 & 66.67 & 33.33 \\
\hline & TOTAL & 74 & 42 & 116 & 63.79 & 36.21 \\
\hline
\end{tabular}

Fuente: Consejo Nacional Electoral - Elecciones 2014

Datos tomados del Documento: Atlas De Genero 2018 
Tabla No 2: Prefectos Provinciales Desagregados por Género.

\begin{tabular}{|r|l|r|r|}
\hline \multirow{2}{*}{ Codigo } & \multicolumn{2}{|c|}{ Provincia } & \multicolumn{2}{c|}{ Prefectos } \\
\cline { 3 - 4 } & & Hombres & Mujeres \\
\hline \hline 2 & AZUAY & 1 & 0 \\
\hline 3 & BOLIVAR & 1 & 0 \\
\hline 4 & CAÑAR & 1 & 0 \\
\hline 5 & CORCHI & 1 & 0 \\
\hline 6 & CHIMBORAXI & 1 & 0 \\
\hline 7 & EL ORO & 1 & 0 \\
\hline 8 & ESMERALDAS & 1 & 1 \\
\hline 9 & GUAYAS & 0 & 0 \\
\hline 10 & IMBABURA & 1 & 0 \\
\hline 11 & LOJA & 1 & 0 \\
\hline 12 & LOS RIOS & 1 & 0 \\
\hline 13 & MANABI & 1 & 0 \\
\hline 14 & MORONA SANTIAGO & 1 & 0 \\
\hline 15 & NAPO & 1 & 0 \\
\hline 16 & PASTAZA & 1 & 0 \\
\hline 17 & PINCHINCHA & 1 & 0 \\
\hline 18 & TUNGURAHUA & 1 & 0 \\
\hline 19 & ZAMORA CHINCHIPE & 1 & 0 \\
\hline 20 & GALAPAGOS & 0 & 0 \\
\hline 21 & SUCUMBIOS & 1 & 0 \\
\hline 22 & ORELLNA & 1 & 0 \\
\hline 23 & STO DGO TSACHILAS & & 0 \\
\hline 24 & SANTA ELENA & 1 & 0 \\
\hline \hline
\end{tabular}

Fuente: Consejo Nacional Electoral - elecciones 2014

Datos Tomados del Documento: "Análisis de Resultados Elecciones 2014" Publicado por CNE. 
Tabla No 3: Alcaldes municipales desagregados por género, a nivel provincial.

\begin{tabular}{|c|c|c|c|c|c|c|}
\hline \multirow{2}{*}{ Codigo } & \multirow{2}{*}{ Provincia } & \multicolumn{5}{|c|}{ Alcaldes Municipales } \\
\hline & & Hombres & Mujeres & Total & \%Hombres & $\%$ Mujeres \\
\hline 1 & AZUAY & 13 & 2 & 15 & 86.67 & 13.33 \\
\hline 2 & BOLIVAR & 7 & 0 & 7 & 100 & 0 \\
\hline 3 & CAÑAR & 7 & 0 & 7 & 100 & 0 \\
\hline 4 & CARCHI & 6 & 0 & 6 & 100 & 0 \\
\hline 5 & COTOPAXI & 7 & 0 & 7 & 100 & 0 \\
\hline 6 & CHIMBORAZO & 10 & 0 & 10 & 100 & 0 \\
\hline 7 & EL ORO & 13 & 1 & 14 & 92.86 & 7.14 \\
\hline 8 & ESMERALDAS & 7 & 0 & 7 & 100 & 0 \\
\hline 9 & GUAYAS & 20 & 5 & 25 & 80 & 20 \\
\hline 10 & IMBABURA & 6 & 0 & 6 & 100 & 0 \\
\hline 11 & LOJA & 14 & 2 & 16 & 87.5 & 12.5 \\
\hline 12 & LOS RIOS & 12 & 1 & 13 & 92.31 & 7.69 \\
\hline 13 & MANABI & 21 & 1 & 22 & 95.45 & 4.55 \\
\hline 14 & MORONA SANTIAGO & 12 & 0 & 12 & 100 & 0 \\
\hline 15 & NAPO & 5 & 0 & 5 & 100 & 0 \\
\hline 16 & PASTAZA & 4 & 0 & 4 & 100 & 0 \\
\hline 17 & PINCHINCHA & 6 & 2 & 8 & 75 & 25 \\
\hline 18 & TUNGURAHUA & 9 & 0 & 9 & 100 & 0 \\
\hline 19 & ZAMORA CHINCHIPE & 9 & 0 & 9 & 100 & 0 \\
\hline 20 & GALAPAGOS & 3 & 0 & 3 & 100 & 0 \\
\hline 21 & SUCUMBIOS & 6 & 1 & 7 & 85.71 & 14.29 \\
\hline 22 & ORELLANA & 3 & 1 & 4 & 75 & 25 \\
\hline 23 & STO DGO TSACHILAS & 2 & 0 & 2 & 100 & 0 \\
\hline 24 & SANTA ELENA & 3 & 0 & 3 & 100 & 0 \\
\hline & 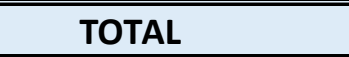 & 205 & 16 & 221 & 2270.5 & 129.5 \\
\hline
\end{tabular}

Fuente: Consejo Nacional Electoral - Elecciones 2014

Datos Tomados del Documento: "Análisis de Resultados Elecciones 2014" Publicado por CNE, Confirmados Con La Página Web de la Asociación de Municipalidades Del Ecuador. 
Tabla No 4: Concejales municipales desagregados por género, a nivel provincial.

\begin{tabular}{|c|c|c|c|c|c|c|c|c|c|c|c|}
\hline \multirow{2}{*}{ Codigo } & \multirow{2}{*}{ Provincia } & \multicolumn{5}{|c|}{ Concejales Urbanos } & \multicolumn{5}{|c|}{ Consejales rurales } \\
\hline & & Hombres & MUJERES & TOTAL & $\%$ Hombres & \%Mujeres & Hombres & Mujeres & Total & \%Hombres & $\%$ Mujeres \\
\hline 1 & AZUAY & 36 & 17 & 53 & 67.92 & 32.08 & 23 & 9 & 32 & 71.88 & 28.13 \\
\hline 2 & BOLIVAR & 18 & 9 & 27 & 66.67 & 33.33 & 9 & 1 & 10 & 90.00 & 10.00 \\
\hline 3 & CAÑAR & 18 & 10 & 28 & 64.29 & 35.71 & 8 & 5 & 13 & 61.54 & 38.46 \\
\hline 4 & CARCHI & 14 & 4 & 18 & 77.78 & 22.22 & 9 & 5 & 14 & 64.29 & 35.71 \\
\hline 5 & СОTOPAXI & 14 & 8 & 22 & 63.64 & 36.36 & 18 & 3 & 21 & 85.71 & 14.29 \\
\hline 6 & CHIMBORAZO & 27 & 9 & 36 & 75.00 & 25.00 & 17 & 3 & 20 & 85.00 & 15.00 \\
\hline 7 & EL ORO & 37 & 23 & 60 & 61.67 & 38.33 & 15 & 7 & 22 & 68.18 & 31.82 \\
\hline 8 & ESMERALDAS & 17 & 5 & 22 & 77.27 & 22.73 & 17 & 4 & 21 & 80.95 & 19.05 \\
\hline 9 & GUAYAS & 83 & 54 & 137 & 60.58 & 39.42 & 20 & 4 & 24 & 83.33 & 16.07 \\
\hline 10 & IMBABURA & 13 & 9 & 22 & 59.09 & 40.91 & 11 & 5 & 16 & 68.75 & 31.25 \\
\hline 11 & LOJA & 37 & 12 & 49 & 75.51 & 24.49 & 25 & 12 & 37 & 67.57 & 32.43 \\
\hline 12 & LOS RIOS & 32 & 27 & 59 & 54.24 & 45.76 & 15 & 5 & 20 & 75.00 & 25.00 \\
\hline 13 & MANABI & 65 & 35 & 100 & 65.00 & 35.00 & 25 & 11 & 36 & 69.44 & 30.56 \\
\hline 14 & MORONA SANTIAGO & 23 & 8 & 31 & 74.19 & 25.81 & 25 & 4 & 29 & 86.21 & 13.79 \\
\hline 15 & NAPO & 13 & 3 & 16 & 81.25 & 18.75 & 7 & 4 & 11 & 63.64 & 36.36 \\
\hline 16 & PASTAZA & 10 & 2 & 12 & 83.33 & 16.67 & 7 & 3 & 10 & 70.00 & 30.00 \\
\hline 17 & PINCHINCHA & 30 & 13 & 43 & 69.77 & 30.23 & 15 & 4 & 19 & 70.95 & 21.05 \\
\hline 18 & TUNGURAHUA & 25 & 11 & 36 & 69.44 & 30.56 & 13 & 6 & 19 & 68.42 & 31.58 \\
\hline 19 & ZAMORA CHINCHIPE & 18 & 8 & 26 & 69.23 & 30.77 & 15 & 4 & 19 & 78.95 & 21.05 \\
\hline 20 & GALAPAGOS & 10 & 2 & 12 & 83.33 & 16.67 & 3 & 0 & 3 & 100.00 & 0.00 \\
\hline 21 & SUCUMBIOS & 11 & 8 & 19 & 57.89 & 42.11 & 15 & 3 & 18 & 83.33 & 16.67 \\
\hline 22 & ORELLNA & 7 & 3 & 10 & 70.00 & 30.00 & 10 & 2 & 12 & 83.33 & 16.67 \\
\hline 23 & STO DGO TSACHILAS & 6 & 9 & 15 & 40.00 & 60.00 & 2 & 1 & 3 & 66.67 & 33.33 \\
\hline 24 & SANTA ELENA & 9 & 5 & 14 & 64.29 & 35.71 & 5 & 4 & 9 & 55.56 & 44.44 \\
\hline & TOTAL & 573 & 294 & 867 & 66.09 & 33.91 & 329 & 109 & 438 & 75.11 & 24.89 \\
\hline
\end{tabular}

Fuente: Consejo Nacional Electoral - Elecciones 2014

Datos Tomados del Documento: "Análisis de Resultados Elecciones 2014" Publicado por CNE, Confirmados Con La Página Web De La Asociación de Municipalidades Del Ecuador. 
Tabla No 5: Vocales de las Juntas Parroquiales Rurales desagregados por género, a nivel provincial.

\begin{tabular}{|c|c|c|c|c|c|c|}
\hline \multirow[b]{2}{*}{ Codigo } & \multirow[b]{2}{*}{ Provincia } & \multicolumn{5}{|c|}{ Vocales juntas Parroquiales rulares } \\
\hline & & Hombres & MUJERES & TOTAL & $\%$ Hombres & \%Mujeres \\
\hline 1 & AZUAY & 227 & 78 & 305 & 74.43 & 25.57 \\
\hline 2 & BOLIVAR & 80 & 15 & 95 & 84.21 & 15.79 \\
\hline 3 & CAÑAR & 97 & 33 & 130 & 74.62 & 25.38 \\
\hline 4 & CARCHI & 97 & 33 & 130 & 74.62 & 25.38 \\
\hline 5 & COTOPAXI & 137 & 28 & 165 & 83.03 & 16.97 \\
\hline 6 & CHIMBORAZO & 165 & 60 & 225 & 73.33 & 26.67 \\
\hline 7 & EL ORO & 176 & 69 & 245 & 71.84 & 28.16 \\
\hline 8 & ESMERALDAS & 204 & 81 & 285 & 71.58 & 28.42 \\
\hline 9 & GUAYAS & 95 & 50 & 145 & 65.52 & 34.48 \\
\hline 10 & IMBABURA & 129 & 51 & 180 & 71.67 & 28.33 \\
\hline 11 & LOJA & 300 & 90 & 390 & 76.62 & 23.08 \\
\hline 12 & LOS RIOS & 52 & 33 & 85 & 71.18 & 38.82 \\
\hline 13 & MANABI & 192 & 73 & 265 & 72.55 & 27.55 \\
\hline 14 & MORONA SANTIAGO & 187 & 43 & 230 & 81.3 & 18.7 \\
\hline 15 & NAPO & 73 & 27 & 100 & 73 & 27 \\
\hline 16 & PASTAZA & 63 & 22 & 85 & 74.12 & 25.88 \\
\hline 17 & PINCHINCHA & 195 & 74 & 269 & 72.49 & 27.51 \\
\hline 18 & TUNGURAHUA & 183 & 37 & 220 & 83.18 & 16.82 \\
\hline 19 & ZAMORA CHINCHIPE & 109 & 31 & 140 & 77.86 & 22.14 \\
\hline 20 & GALAPAGOS & 17 & 8 & 25 & 68 & 32 \\
\hline 21 & SUCUMBIOS & 100 & 35 & 135 & 74.07 & 25.93 \\
\hline 22 & ORELLNA & 111 & 29 & 140 & 79.29 & 20.71 \\
\hline 23 & STO DGO TSACHILAS & 39 & 11 & 50 & 28 & 22 \\
\hline 24 & SANTA ELENA & 28 & 12 & 40 & 70 & 30 \\
\hline & TOTAL & 3056 & 1023 & 4079 & 74.92 & 25.08 \\
\hline
\end{tabular}

Fuente: Consejo Nacional Electoral - Elecciones 2014

Datos Tomados del Documento: "Análisis de Resultados Elecciones 2014" Publicado por CNE 